\title{
Response cost and time-place discrimination by rats in maze tasks
}

\author{
DAVID R. WIDMAN and DOUGLAS GORDON \\ Kalamazoo College, Kalamazoo, Michigan \\ and \\ WILLIAM TIMBERLAKE \\ Indiana University, Bloomington, Indiana
}

\begin{abstract}
Time-place discrimination has been shown reliably in several avian and insect species, but only occasionally in rats and fish. In the present experiments, we explored the effects of response cost on time-place discrimination by rats. In the first experiment, we increased the cost of making a choice and the cost of recovering from a wrong choice in two types of maze, a radial arm and a vertical maze. In the radial arm maze, we found only general place preference, whereas in the vertical maze, we obtained evidence of time-place discrimination. In the second experiment, we found that the proportion of rats showing time-place discrimination increased with the height and, therefore, the response cost of the vertical maze. These results suggest that rats do not automatically store and/or retrieve the time and place of reward events but that response cost is an important trigger for time-place discrimination.
\end{abstract}

In recent years, how an organism learns about food resources that vary in both time and place has come under experimental scrutiny (e.g., Biebach, Gordijn, \& Krebs, 1989; Reebs, 1993; Wilkie, Saksida, Samson, \& Lee, 1994). Wilkie (1995) described a theory of time-place learning (based on Gallistel, 1990) in which biologically important events trigger automatic memory storage of a time-place-event code. According to this theory, a biologically significant event (such as the finding of food by a hungry animal) triggers the recording of three memories: (1) the time the event occurred, (2) the spatial location where the event occurred, and (3) the exact nature of the event itself. These codes are transferred into the animal's long-term memory for later retrieval. When the organism again experiences a need for a particular resource, it presumably scans its memory for the nearest location of that resource at the current time.

Several species have demonstrated time-place discriminations (e.g., bees: Gould, 1987; ants: Harrison \& Breed, 1987; fish: Reebs, 1996); however, most work has been done with birds. Krebs and colleagues generated an extensive body of research on time-place discrimination

Experiments $1 \mathrm{~A}$ and $1 \mathrm{~B}$ were presented at the 69 th Annual Meeting of the Eastern Psychological Association, February 1998. The second experiment served as partial fulfillment of the Senior Individualized Project at Kalamazoo College for D.G. Portions of this research were supported by Grant MH 37892 from the National Institutes of Health awarded to W.T. and by funds for Student-Faculty Research Projects from the Kalamazoo College Faculty Development Committee awarded to D.R.W. The authors thank Jeannette Morgan and Thomas Stibitz for their help running the experiments and Gary Lucas for his comments on an earlier draft. Correspondence concerning this article should be addressed to D. R. Widman, Department of Psychology, Juniata College, Huntingdon, PA 16652 (e-mail: widman @juniata.edu). in birds. In a typical procedure, garden warblers lived in an aviary consisting of a central living area connected to four feeding areas (Biebach et al., 1989). Each of the four areas contained a food source for a 3-h block during the 12-h daily training sessions. If the birds selected an incorrect feeding area for the time of day, they were denied access to all the feeding areas for $280 \mathrm{sec}$. The same pattern of food availability was maintained until the birds reached a criterion of $75 \%$ of entries to the correct area during the correct time. They were then tested with food available for the entire $12-\mathrm{h}$ session in every room. Despite freely available food, they maintained the daily spatial and temporal patterns of feeding established during training. Further studies revealed that these birds were most probably using circadian oscillators to time the availability of food (Biebach, Falk, \& Krebs, 1991; Krebs \& Biebach, 1989).

More recently, Wilkie and colleagues studied timeplace discriminations in pigeons (Saksida \& Wilkie, 1994; Wilkie et al., 1994) and rats (Carr \& Wilkie, 1997, 1998). The animals were placed once in the morning and once in the afternoon into operant chambers with response manipulanda and food hoppers on each of the four walls. The animals were rewarded for responding at one wall in the morning and a second wall in the afternoon. Both the pigeons and the rats formed time-place discriminations, responding to the appropriate manipulanda during the different feedings, although the rats and the pigeons apparently used different mechanisms (Carr \& Wilkie, 1997, 1998).

Despite the apparent widespread occurrence of timeplace learning, work with rats has actually shown mixed results. Carr, Wilkie, and colleagues, using the operant chamber procedure described above, reliably find time- 
place discrimination in rats (Carr, Tan, \& Wilkie, 1999; Carr \& Wilkie, 1997, 1998, 1999). Mistlberger, de Groot, Bossert, and Marchant (1996) also found time-place discriminations in rats, using a procedure in which rats had to select the correct arm of a T-maze and then barpress to obtain food. Boulos and Logothetis (1990), on the other hand, using easier access to food and a longer total session time, did not. The latter authors trained rats to obtain food by pressing one of two levers located on opposite sides of a cylindrical apparatus during a 1- or 2-h block of time and the opposite lever during a different $1-$ or $2-\mathrm{h}$ block. Two of the 4 rats showed more anticipatory pressing to the time-appropriate lever even when no food was presented for up to 5 days. The other 2 rats displayed, instead, reliable preferences for one lever over the other.

Previous work in our laboratory also failed to show reliable time-place discrimination (White \& Timberlake, 1990). Rats were exposed to a three-arm radial maze at three different times of day. Food $(2 \mathrm{~g})$ was available at the end of a different arm at each of the three different times. The rats here also failed to form time-place discriminations. A follow-up pilot study showed a similar absence of time-place discrimination in a two-choice apparatus more similar to that of Boulos and Logothetis (1990).

Boulos and Logothetis (1990) suggested that the relatively poor discrimination performance of their rats might have been due to the small amount of cost in time and/or energy required to switch between the alternatives. In addition, the reinforcement schedule in effect at each location may have been too rich. Once reinforcement was available, continuous reinforcement CRF schedules were in effect at both locations and times. In either case, low response cost may have been responsible for the failure to form time-place discriminations. Our data (White \& Timberlake, 1990) are consistent with the response cost argument. The only consequence for initially selecting an incorrect location was a slightly longer trip to the correct location, a very small penalty.

Reebs (1993) also attributed a failure to find timeplace discriminations in convict cichlid fish to low response cost. After training with a signal $1 \mathrm{~min}$ prior to food presentation, the fish were offered food following the signal in a corner of the aquarium that depended on time of day. Instead of shifting their reaction to the food signal as a function of time of day, the fish showed a preference for going to the morning corner. Reebs (1993) suggested that the cost of traveling from one corner of the aquarium to the other was so minimal that forming the time-place discrimination was not important for the fish in foraging for food.

The purpose of the present experiments was to examine the effect of response cost in a multilocation maze environment on the formation of time-place discriminations by rats. We chose to focus on a multilocation maze environment for several reasons. First, the laboratory problem would seem more readily related to the rat's for- aging ecology if there were multiple alternative locations that were spatially separated by a greater distance than the different walls of an operant chamber. Second, finding time-place discrimination without imposing an operant manipulation requirement should increase the generality of time-place learning in the laboratory. Third, using a four-alternative spatial choice maze provided a closer comparison with the spatial choice task imposed on songbirds (Biebach et al., 1991; Biebach et al., 1989; Krebs \& Biebach, 1989).

The purpose of Experiments 1A and 1B was to develop a maze task that would reliably produce time-place discriminations in rats. Experiment $1 \mathrm{~A}$ used a four-arm radial maze, whereas Experiment $1 \mathrm{~B}$ used a vertical maze. After establishing such a discrimination, the purpose of the second experiment was to test for a functional relation between response cost and time-place discrimination.

\section{EXPERIMENT 1A}

In Experiment $1 \mathrm{~A}$, we extended the radial arm maze procedure used by White and Timberlake (1990). In this case, we used procedures more like those of Carr and Wilkie (1997), using four arms with two baited arms at two different times of day. In addition, we increased the response cost at two locations in the search chain: choice cost and recovery cost. Of course, response cost typically involves dimensions of both time and effort, but this experiment emphasized the temporal location of cost in relation to reward. Choice cost refers to the cost of completing a response necessary to discover whether food is present at a particular location. The specific choice costs employed were patterned after two procedures developed by Roberts and Ilersich (1989). The first involved the use of a pyramidal barricade at the start of each arm, approximately $13.3 \mathrm{~cm}$ high. The second involved placing weighted covers on the food cups. Roberts and Ilersich found that these methods significantly increased the time required to obtain the total reinforcement available on a four-arm radial arm maze, as compared with conditions of only barricades, only covers, or no barricades or covers. In addition, they reported that the presence of barricades produced an increase in patch residence time. Similar changes in responding have been predicted and observed in other situations where the cost of patch selection was increased (Mellgren, Misasi, \& Brown, 1984; Ydenberg, 1984).

The recovery cost refers to the cost of returning to the point at which the next choice can be initiated. For example, Krebs and colleagues (Biebach et al., 1991; Biebach et al., 1989; Krebs \& Biebach, 1989) employed a long temporal recovery cost in their work. If the bird chose the wrong food area, it had to wait $280 \mathrm{sec}$ for another choice opportunity. Such an increase in delay to reward has been suggested to be a central component of cost in operant foraging situations (Fantino \& Abarca, 1985). 
Following these experiments, we imposed a home cage time-out of $15 \mathrm{~min}$ when animals made an incorrect choice before they were returned to the choice point of the maze.

\section{Method}

Subjects. Five female, Sprague-Dawley rats born and raised in the Indiana University Psychology rat colony served as subjects. They were $90-120$ days of age at the beginning of training. The rats were deprived to $80 \%$ of their body weight and allowed $20 \mathrm{~g}$ of maintenance food, as well as an ad-lib access to water. The colony was on a 12:12 h light:dark cycle, with lights on at 0700 and lights off at 1900 .

Apparatus. A four-arm radial arm maze was used to train the time-place discriminations. The maze was placed in the same room where the animals were housed. An opaque curtain that extended from the floor to the ceiling separated the maze area from the living area. There were several room cues that allowed spatial discrimination. These included a two-way mirror on the east wall, a door on the south wall, a shelf with recording equipment and a separately mounted video camera on the north wall, and the dividing curtain on the west wall. In addition, the experimenter sat in a consistent location, on the south wall, throughout the experiment. These cues were kept constant throughout the experiment.

The maze was constructed of wood and painted gray. It was elevated $67 \mathrm{~cm}$ off the floor. The central platform of the maze was an octagon measuring $34 \mathrm{~cm}$ in diameter. Four arms extended from the maze at $90^{\circ}$ angles. These arms each measured $70 \mathrm{~cm}$ long. The arms were enclosed by side walls. The first $32 \mathrm{~cm}$ of the left sidewall was $12 \mathrm{~cm}$ high. The remaining left sidewall and the entire right sidewall were $3 \mathrm{~cm}$ high. Wooden obstacles were placed in each arm. These were constructed from three lengths of $2 \times 4$ in. boards attached in a pyramid form. It measured $13.3 \mathrm{~cm}$ high. A nonskid rubber mat was attached to the bottom of the structure to prevent movement along the surface of the arm and to enhance stability. Located $1.5 \mathrm{~cm}$ from the far end of each arm was a metal feeding cup. These feeding cups measured $8 \mathrm{~cm}$ in diameter and $2.5 \mathrm{~cm}$ deep. A wooden lid that measured $8.5 \mathrm{~cm}$ in diameter and $0.5 \mathrm{~cm}$ tall covered each cup. Each lid was constructed so that it fit into the food cup with a $0.5-\mathrm{cm}$ lip extending over the edge of the food cup. Fifteen $45-\mathrm{mg}$ food pellets (Noyes Formula P) were placed within the cups.

Procedure. The procedure consisted of two phases: pretraining and discrimination training. During both phases, the subjects were placed into the apparatus individually.

Pretraining. Pretraining consisted of exposure to the maze and gradual training to climb over the obstacles and remove the food cup lids. The animals were first exposed to the maze without obstacles or lids and allowed to find food in the cups. As the animals became proficient at finding the food, increasingly larger obstacles were placed in the arms, until animals were climbing over obstacles of the same size as those used in training. Simultaneously, the food cup lids were placed over the food cups, first simply partially covering the food cups; then, coverage was progressively increased until the animals were finding the food with the lids in the training position. Once the animals were climbing the full obstacles and removing lids in their full position, discrimination training began.

Discrimination training. On the first day of discrimination training, each animal was randomly assigned a morning arm location and an afternoon arm location. There were two constraints placed on this assignment. First, no animal could have the same arm for both the morning and the afternoon times. Second, each arm served as a morning arm and an afternoon arm at least once. Food was located on the morning arm only during the morning session and on the afternoon arm only during the afternoon session.

The animals were placed singly onto the central platform each session. They were allowed 5 min to make a selection. A selection was defined as the removal of a food cup lid. If an animal failed to make a selection or made an incorrect selection, it was removed from the maze and returned to its home cage. After $15 \mathrm{~min}$, it was again placed onto the central platform and allowed another $5 \mathrm{~min}$ to make a selection. If it again failed to make a selection or made an incorrect selection, it was returned to its home cage for a second 15-min period. Following this period, the animal was placed onto the central platform and allowed to forage on the maze until it found the food. The animals received two such sessions, one $2.5 \mathrm{~h}$ after lights on and the other $6 \mathrm{~h}$ later, for 32 days. On the final 5 days, a second measure was recorded, the location of the first food cup inspection. Inspection of a food cup was defined as sniffing at the lid and food cup without removing the lid.

\section{Results and Discussion}

A binomial distribution was used to determine the statistical significance of location selection during the last 6 days of the conditioning phase (27-32). Because there was a one in four chance of an animal randomly selecting the correct location on its first choice, the null hypothesis stated that the probability of a correct choice selected at random was .25. Thus, in order for an animal to select significantly different from chance $(p<.05)$, the animal had to select correctly on 4 of the 6 days for both daily sessions.

Figure 1 presents the choices for each animal for both the morning (upper panel) and the afternoon (lower panel) sessions. As can be seen in Figure 1, Rats 1-1 and 1-5 made a significant number of correct selections for both the morning and the afternoon sessions. Both Rats 1-3 and 1-4 made a significant number of selections to the correct arm in the morning but also made a significant number of selections to the morning location during the afternoon sessions. Finally, Rat 1-2 did not reveal any significant pattern of selections for either the morning or the afternoon sessions.

This pattern of results suggests that 2 animals formed a time-place discrimination, whereas 2 others formed preferences for one location over the other (similar to the results of Boulos \& Logothetis, 1990). However, closer examination of the inspection (sniffing) data added during the final 5 days of the experiment revealed a different result (recall that choice was defined as removing the lid to the food cup and inspection was defined as merely approaching it). Significance for these data, using the binomial distribution and a null hypothesis of $25 \%$ random chance, requires four similar choices out of five. Rat 1 1 tended to first inspect the afternoon arm in the morning and correctly first inspect the afternoon arm in the afternoon. Rat $1-5$, on the other hand, tended to significantly inspect the morning arm first in the morning but did not show a reliable pattern of first inspections in the afternoon. The pattern of first inspections for Rats 1-2, 1-3, and 1-4 did not differ from their respective patterns of selections.

The results of this experiment indicate that 4 of 5 animals formed clear preferences for one location and inspected (sniffed) that location first. If no food was present, the rats then checked the other location where food had been found in the past. One animal did not form any 

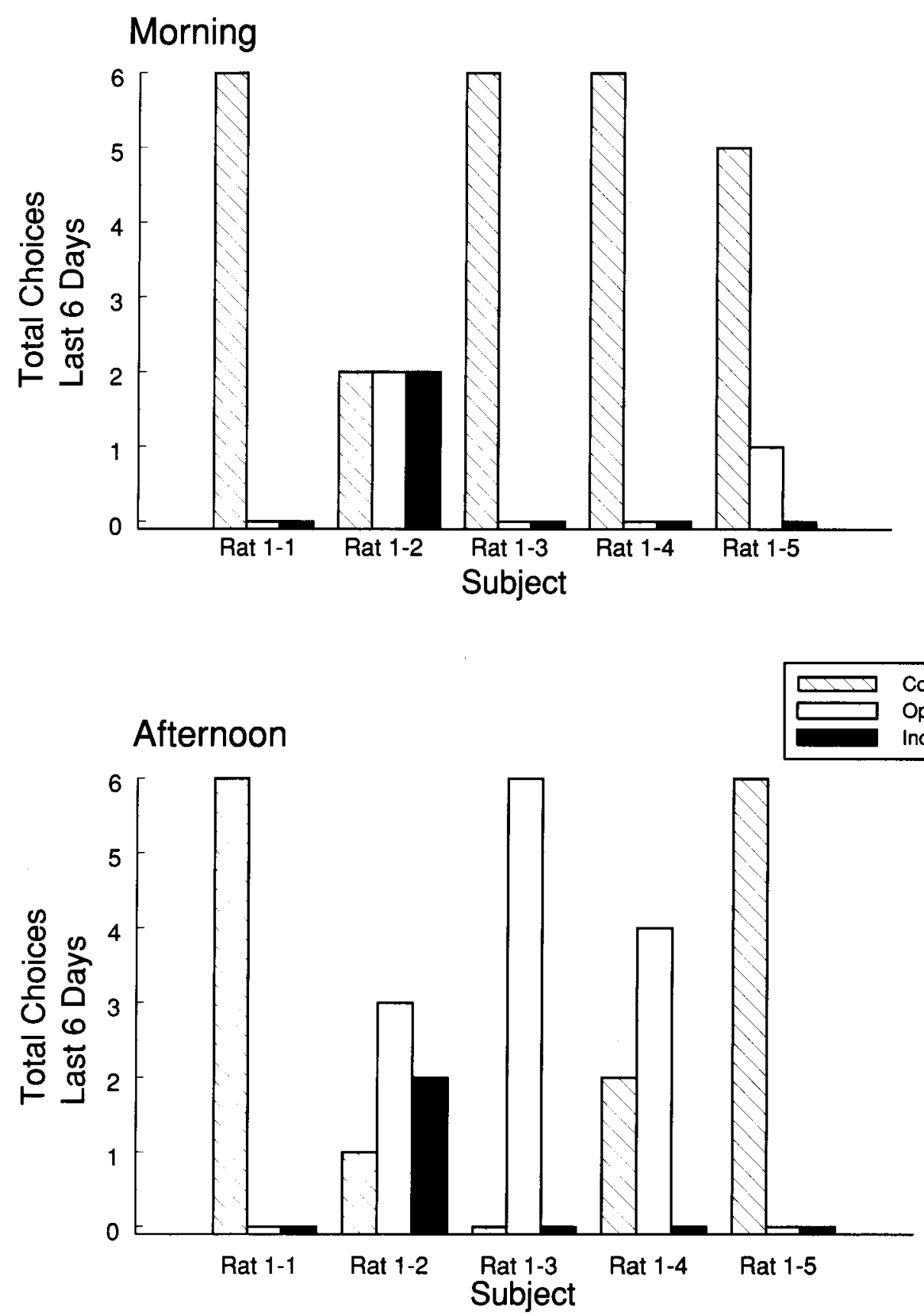

Figure 1. Total number of correct, opposite, and incorrect choices for each animal from the last 6 days of Experiment 1A. The upper panel shows choices from the morning trial, and the lower panel shows choices from the afternoon trial. Correct choices are made to the location baited for the current daily test trial. Opposite choices are made to the location baited for the other daily test trial. Incorrect choices are made to locations that are never baited.

clear pattern of behavior, neither a time-place discrimination nor a preference for one location. In short, no animal appeared to form a time-place discrimination.

There are several possible explanations for this failure. First, it is possible that rats do not form time-place discriminations when tested on mazes but form place preferences instead. Alternatively, it may be that there was insufficient training for time-place discriminations to develop. This alternative seemed unlikely, since most of the animals showed relatively stable choice patterns during the final 10 days of testing, concentrating their choices on the two arms where food was located. Finally, it is possible that although our response cost procedures were sufficient to produce changes in behavior in rats (Roberts \& Ilersich, 1989) and time-place discrimination in birds (Biebach et al., 1991; Biebach et al., 1989), 
they were not sufficient to encourage rats to form timeplace discriminations. Of these possibilities, we chose the issue of response cost to investigate further.

\section{EXPERIMENT 1B}

Since we did not obtain time-place discrimination in Experiment 1A, despite adding temporal response costs, the next experiment focused on the effort dimension of response cost. The purpose of this experiment was threefold. First, we wanted to prevent rats from using odor to detect the presence of food prior to making their choice. The ability of some rats in Experiment $1 \mathrm{~A}$ to sniff the weighted lids and correctly reject cups with no food indicated that olfaction can be an important confound in tests of time-place discrimination. Second, we wanted to continue to use well-separated spatial choices to make contact with foraging ecology and the data from birds. Third, we wanted to ensure that the rats perceived the choice and recovery response costs as effortful.

To satisfy these requirements, we used a vertical maze similar to that used by Mellgren and colleagues (Mellgren \& Brown, 1988; Mellgren et al., 1984). The vertical maze consisted of four towers constructed of $2 \times$ 4 in. wall studs placed on end in an open field. Large nails were driven into one side of each tower to form a ladder, allowing rats to climb and descend. Platforms containing food wells were attached to the towers at a height of $152.4 \mathrm{~cm}$ above the floor. It was assumed that both the choice cost of climbing a tower and the recovery cost of descending a tower would be considerably higher than the choice and recovery costs in the four-arm radial maze in Experiment 1A. Using an apparatus similar to this, Mellgren et al. (1984) demonstrated that rats performed very close to the choice predictions of optimality theory at high search costs. In the present experiment, food was present on the platform of one tower in the morning and a different tower in the afternoon.

\section{Method}

Subjects. Six male, Sprague-Dawley rats were obtained at 80 days of age from Harlan Sprague-Dawley in Indianapolis. They were allowed 5 days to acclimate to the colony prior to training. The rats were then deprived to $80 \%$ of their body weight and were allowed $20 \mathrm{~g}$ of maintenance food, as well as ad-lib access to water. The colony was maintained on a 12:12-h light:dark cycle, with lights on at 0500 and lights off at 1700 . The discrimination training phase began when the animals were 106 days of age.

Apparatus. A vertical maze was used for this experiment. It consisted of an open field with four towers extending vertically from the floor. The floor and walls of the apparatus were built with 1.3cm-thick plywood. The apparatus measured $121.9 \times 121.9 \mathrm{~cm}$, with walls standing $27.9 \mathrm{~cm}$ high. All the surfaces were painted with light gray, latex enamel, flat finish paint. The towers, constructed of pinewood wall studs, measuring $5.1 \times 10.2 \times 213.4 \mathrm{~cm}$, were placed $38.1 \mathrm{~cm}$ from each corner inside the open field. Twenty-five 12-penny $8.3-\mathrm{cm}$ nails were placed approximately $3.2 \mathrm{~cm}$ into the wood every $7.6 \mathrm{~cm}$ along the same narrow side of each tower. These nails served as the means by which the animals climbed the towers. For additional support, four $2.5 \times 5.1 \times 60.7 \mathrm{~cm}$ pieces of pinewood connected the towers at the top. Platforms, constructed of pinewood and measuring $2.5 \times 10.2 \times 17.8 \mathrm{~cm}$, were connected to each tower. Each platform was capable of being secured so that its surface was at any of the following heights from the floor of the open field: $10.2,15.3,30.5,45.7,61.0,76.2,91.4,121.9$, $152.4,182.9$, and $195.6 \mathrm{~cm}$. All the platforms faced outside of the apparatus, with no two platforms facing in the same direction. Depressions, carved into the end of each platform, served as food cups.

The vertical maze was placed into a room that contained several room cues for spatial discrimination. Along the south wall was a large glass cabinet with miscellaneous equipment visible within the cabinet. There was a rack of electromechanical equipment, a video camera, and a floor safe on the west wall. On the north wall, there was a built-in cupboard with miscellaneous equipment on it. The door to the room was located cn the east wall, as well as a shelf with hardware on it. After placing the animals in the maze, the experimenter sat on a chair located in the southwest corner. These cues were kept constant throughout the entire experiment.

Procedure. The procedure for this experiment consisted of two phases: Pretraining and discrimination training. During both phases, the animals were placed into the apparatus individually.

Pretraining. The purpose of the pretraining sessions was to train the subjects to climb the towers of the apparatus. Training lasted 21 days. Pretraining began with the platforms at their lowest position $(10.2 \mathrm{~cm})$ and five food pellets $(45 \mathrm{mg}$, Noyes Precision, Formula $P$ ) placed both in the depressions of each platform and on the floor directly beneath each platform. Once each animal had acclimated to the surroundings and had eaten all of the pellets on two different occasions, the food from the floor was removed, and the platforms were raised to the second height $(15.2 \mathrm{~cm})$. As was the case at the initial height, the animals could eat the five pellets without having to climb the nails. However, at the third height $(30.5 \mathrm{~cm})$, it was necessary for the animals to learn to climb in order to attain the reward. The animals were placed on the nail closest to the platform until they were able to travel from nail to platform. They were then lowered to the nail directly below the platform until they again moved easily from that location to the platform. The platforms were gradually raised to new heights as the animals gained competence in climbing the towers. By the seventh height $(91.4 \mathrm{~cm})$, the animals were no longer placed on the nails but, instead, on the floor at the base of each tower. From this point, the platforms were raised to the next level, once all the animals had accessed each of the four platforms on two separate training sessions. At each of these training heights, the animals were removed from the platform after consuming the food and placed at the base of the next tower.

Once the animals had successfully acquired food at the sixth level, they were no longer removed from the platform but were, instead, required to climb down the towers in order to access another tower. At the eighth height $(121.9 \mathrm{~cm})$, the animals ceased climbing the towers. The reward was doubled from 5 pellets to 10 , and the animals' previous climbing behavior was restored. At the ninth height $(152.4 \mathrm{~cm})$, the animals were no longer placed at the base of each tower but, rather, in the middle of the apparatus. Once the subjects had climbed up and down each tower unassisted at the ninth height $(152.4 \mathrm{~cm})$, as well as traveled from tower to tower without any assistance from the experimenter on two consecutive training occasions, the pretraining phase ended, and discrimination training began.

Time-place discrimination training. Each animal was randomly assigned two baited towers, one for the morning session and one for the afternoon session. The same constraints were placed on this assignment as in the previous experiment: No animal could have the same tower for both the morning and the afternoon ses- 
sions, and each tower served as a morning and an afternoon tower at least once. Fifteen food pellets were placed on the appropriate tower at the appropriate time of day; no other food was available in the apparatus. The platforms remained at the final height of the training phase $(152.4 \mathrm{~cm})$ for the entire conditioning phase. The conditioning phase lasted 25 days. If an animal did not show a significant pattern of selections for both the morning and the afternoon time periods during the final 6 days of conditioning, it was allowed 5 more days of conditioning. If a significant pattern of selections was still not observed, the animals received an additional 5 days of conditioning. Conditioning for all the animals ended following this additional amount of conditioning. Only 1 animal required one additional set of conditioning trials.

Morning sessions began $3.5 \mathrm{~h}$ after lights on, and the afternoon sessions began $5 \mathrm{~h}$ following the morning session, $8.5 \mathrm{~h}$ after lights on. Before each session the floor of the apparatus, as well as each platform, was cleaned with a damp sponge and a cleaning solution containing a 10:1 ratio of water to a lemon disinfectant (Calpro Institutional, 5180). The animals were placed in the center of the apparatus, facing the same direction each time. For videotaping purposes, the experimenter would call out loud the identification of the animal and the session (i.e., "Rat Number 3, Day 7, PM.") and then sit in the chair. An attempt was recorded each time an animal reached the fourth nail $(30.5 \mathrm{~cm})$ with its hind legs. A climb was recorded each time a subject successfully reached a platform. Only the data from the animals' climbs are reported, since there were only four occasions on which an animal made an attempt without reaching the platform. Once the animal had reached the platform with the food and had finished eating, they were removed from the platform without being allowed to climb down the tower. If an animal did not make an attempt within $10 \mathrm{~min}$, it was placed at the bottom of the correct tower for that time until it climbed. Similarly, if an animal climbed a tower that was not baited and did not climb down after $10 \mathrm{~min}$, it was placed onto the nails directly next to the platform until it climbed down. Only 1 animal required this assistance on three separate occasions early in the discrimination training phase.

\section{Results and Discussion}

The significance of the last 6 days' choices was determined in the same manner as in Experiment 1. Figure 2 displays these choices for each animal. The selections of Rats 2-3, 2-4, 2-5, and 2-6 were each significantly greater than chance for both daily sessions; these rats showed clear time-place discriminations. Rat 2-3 required an additional set of sessions to obtain a consistent pattern. Rats 2-1 and 2-2, however, showed a different pattern of selections. On the final 6 days of conditioning, Rats 2-1 and 2-2 displayed significant preferences for the afternoon location, regardless of time of session.

The results of this experiment support the hypothesis that response cost influences the formation of timeplace discriminations. With the presumed higher total cost in the second experiment, $66 \%$ of the animals showed time-place discriminations, as compared with none of the rats in the first experiment. Interestingly, informal observations indicated that the rats had more difficulty climbing down than up, taking longer and often jumping to the floor rather than climbing down the last few nails. The rats rarely fell (and even more rarely after the first few training days) and, after training, showed no overt hesitation in climbing the towers. However, the apparent difficulty of climbing down raises the possibility that mild fear could have contributed to response cost.

\section{EXPERIMENT 2}

The purpose of the second experiment was to vary response cost on the vertical maze from low to high, thereby allowing a direct assessment of the effects of response cost on the formation of time-place discriminations. Although Experiment 1B, when compared with Experiment $1 \mathrm{~A}$, strongly suggested that effort is an important determinant of time-place discriminations, we have no direct evidence that response cost was responsible, rather than some other unique quality of the vertical maze. In the present experiment, three groups of rats experienced different levels of response cost, defined in terms of platform height. For one group, the platform was placed at the $45.7-\mathrm{cm}$ height. A second group was required to climb to the same height as that used in Experiment $1 \mathrm{~B}$ $(152.4 \mathrm{~cm})$. The final group was required to climb to a higher level $(195.6 \mathrm{~cm})$. We predicted that if increased response effort was responsible for the increased formation of time-place discriminations observed in the second experiment, the number of animals forming time-place discriminations in the present experiment would increase with the height of the platforms.

\section{Method}

Subjects. Fourteen male Sprague-Dawley rats, obtained at 80 days of age from Harlan Sprague-Dawley, served as subjects. They were allowed 5 days to acclimate to the colony conditions prior to training. The rats were then deprived to $80 \%$ of their body weight and were allowed $20 \mathrm{~g}$ of maintenance food and ad-lib access to water. The colony was on a 12:12-h light:dark cycle, with lights on at 1200 and lights off at 000 . The rats were approximately 90 days of age when training began.

Apparatus. The apparatus was the same as that used in the previous experiment and was placed in the same room.

Procedure. The procedure was identical to that in the previous experiment, with several exceptions. First, the animals were divided into three groups. Five rats were assigned to the low platform group $(45.7 \mathrm{~cm}), 5$ animals were assigned to the intermediate height platform group $(152.4 \mathrm{~cm})$, and 4 animals were assigned to the upper platform group $(195.6 \mathrm{~cm})$. All assignment was done randomly. Second, all the animals received pretraining, as in the previous experiment, to the $152.4-\mathrm{cm}$ level, with the exception that ten $45-\mathrm{mg}$ pellets were placed on the platforms at all heights.

Following pretraining to climb up and down at the $152.4-\mathrm{cm}$ height, the platforms were moved to the animals' respective conditioning height $(45.7,152.4$, and $195.6 \mathrm{~cm})$. Pretraining continued at the eventual conditioning heights until all the animals reached the same criterion as that in Experiment 1B: climb up and down each tower, as well as travel from tower to tower unassisted, on two consecutive training trails. Finally, seventeen $45-\mathrm{mg}$ Noyes Formula $P$ pellets were placed in each food cup during this final pretraining and the discrimination training phase, instead of the 15 used in the prior experiment. Seventeen pellets were used in an effort to compensate for the increase in height of the highest platform and, thus, head off any tendency of the rats to stop climbing, as occurred in the training phase of Experiment 1B. The results indicated that the in- 

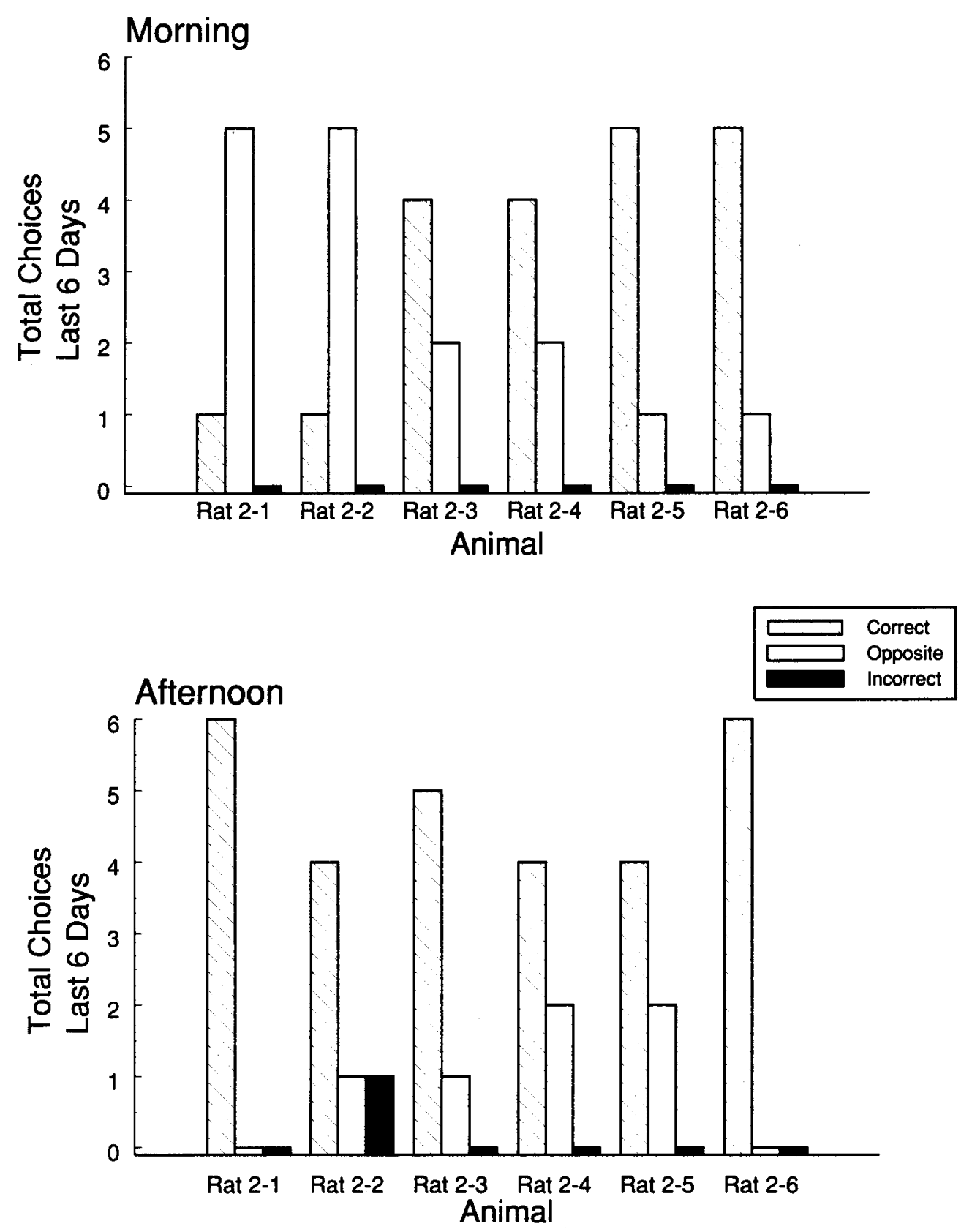

Figure 2. Total number of correct, opposite, and incorrect choices for each animal from the last 6 days of Experiment 1B. The upper panel shows choices from the morning time, and the lower panel shows choices from the afternoon time. Correct choices are made to the location baited for the current daily test trial. Opposite choices are made to the location baited for the other daily test trial. Incorrect choices are made to locations that are never baited.

crease from 15 to 17 pellets did not change performance at the previous platform height.

Discrimination training consisted of 25 days of conditioning and two additional sets of 5 days of discrimination training, if needed. Five rats required the first set of 5 additional days (one from the lowest height, three from the intermediate height, and one from the tallest height). Three of these animals also required the second set of
5 days of additional training (one from the lowest height and two from the intermediate height).

\section{Results and Discussion}

Choices are presented in Figure 3 for those animals that climbed to the lower height, Figure 4 for the animals 

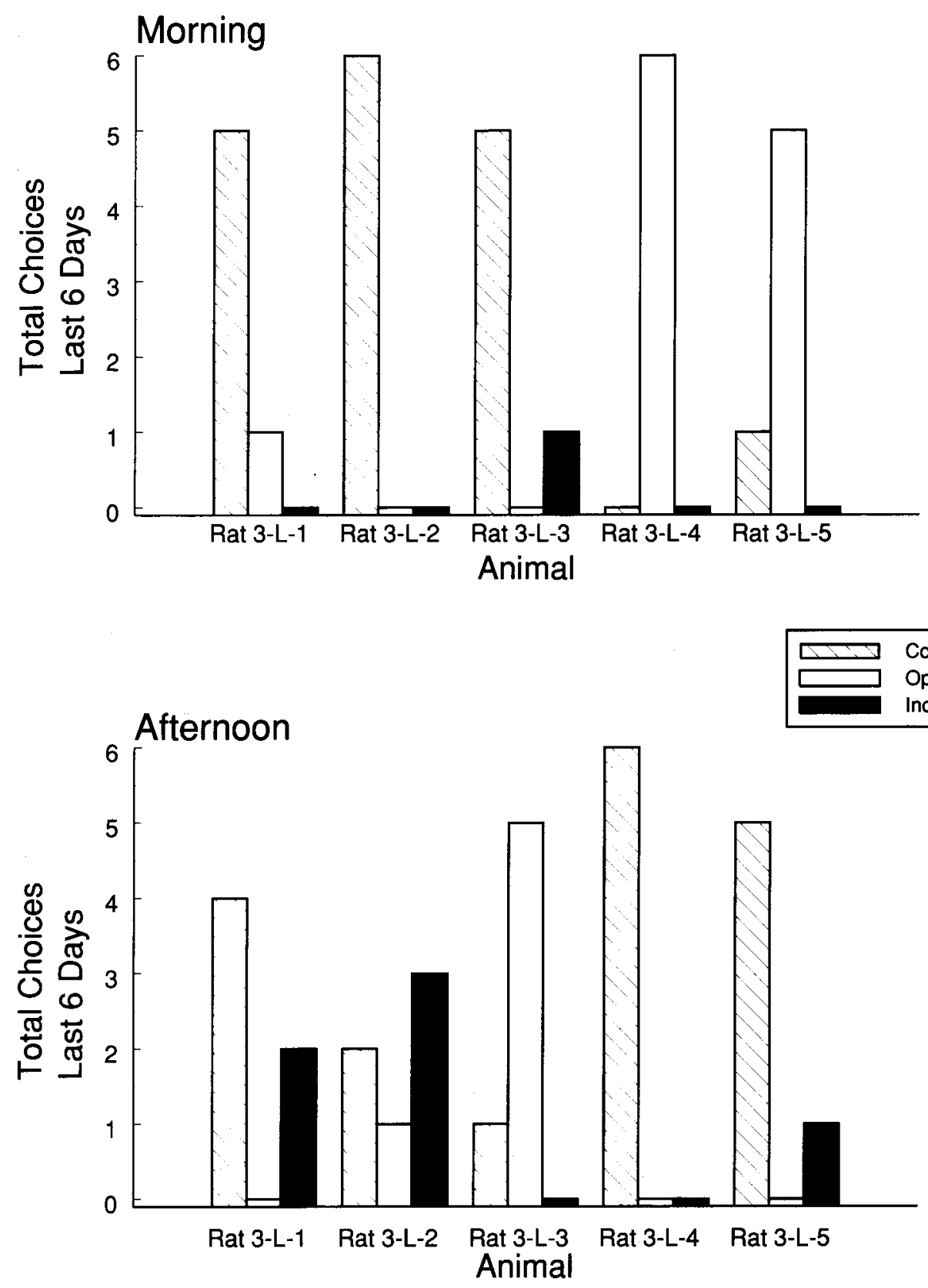

Figure 3. Total number of correct, opposite, and incorrect choices for each animal from the last 6 days of animals that climbed to the lowest level in Experiment 2. The upper panel shows choices from the morning time, and the lower panel shows choices from the afternoon time. Correct choices are made to the location baited for the current daily test trial. Opposite choices are made to the location baited for the other daily test trial. Incorrect choices are made to locations that are never baited.

that climbed to the intermediate height, and Figure 5 for the tallest height. Figure 3 shows that 3 of the 5 rats (Rats 3-L-3, 3-L-4, and 3-L-5) that climbed to the lowest height formed location preferences for either the morning or the evening location. Rat 3-L-1 in the lowest height group formed a time-place discrimination, correctly selecting the morning location in the morning and the afternoon location in the afternoon. Finally, Rat 3-L-2 showed a significant number of correct selections for the morning session but no significant pattern of selections in the afternoon. Figure 4 shows that 2 of the 5 animals that climbed to the intermediate height formed preferences for the afternoon location (Rats 3-M-1 and 3-M-2). The remainder of the animals in the intermediate group formed time-place discriminations (Rats 3-M-3, 3-M-4, and 3-M-5). Finally, Figure 5 shows that all of the ani- 

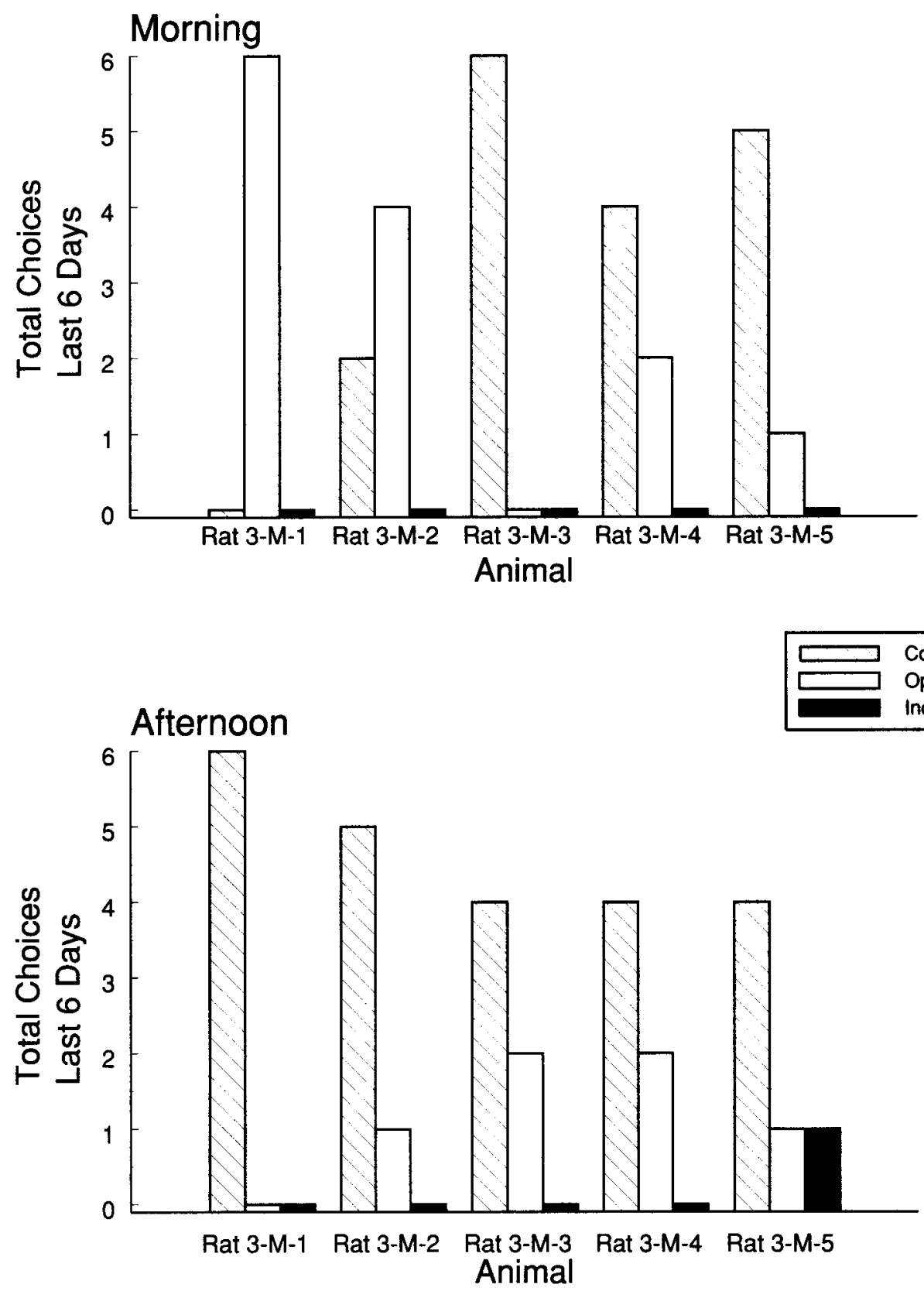

\begin{abstract}
Figure 4. Total number of correct, opposite, and incorrect choices for each animal from the last 6 days of animals that climbed to the intermediate level in Experiment 2. The upper panel shows choices from the morning time, and the lower panel shows choices from the afternoon time. Correct choices are made to the location baited for the current daily test trial. Opposite choices are made to the location baited for the other daily test trial. Incorrect choices are made to locations that are never baited.
\end{abstract}

mals required to climb to the highest platform formed time-place discriminations.

To test the effect of platform height, a JonckheereTerpstra distribution-free test for ordered alternatives (Hollander \& Wolfe, 1973) was performed on the number of sessions each animal selected the correct location for both time periods in the final 6 days of conditioning. This test revealed a significant effect of height on the number of correct selections $[J(5,5,4)=57, p<.05]$, with there being more correct selections at the greater heights. The same test was also performed on the number of selections for the opposite time location-that is, the number of times the animals selected the afternoon location in the morning and the number of times the animals selected the morning location in the afternoon. This test also revealed a significant effect of height $[J(5,5,4)=$ 

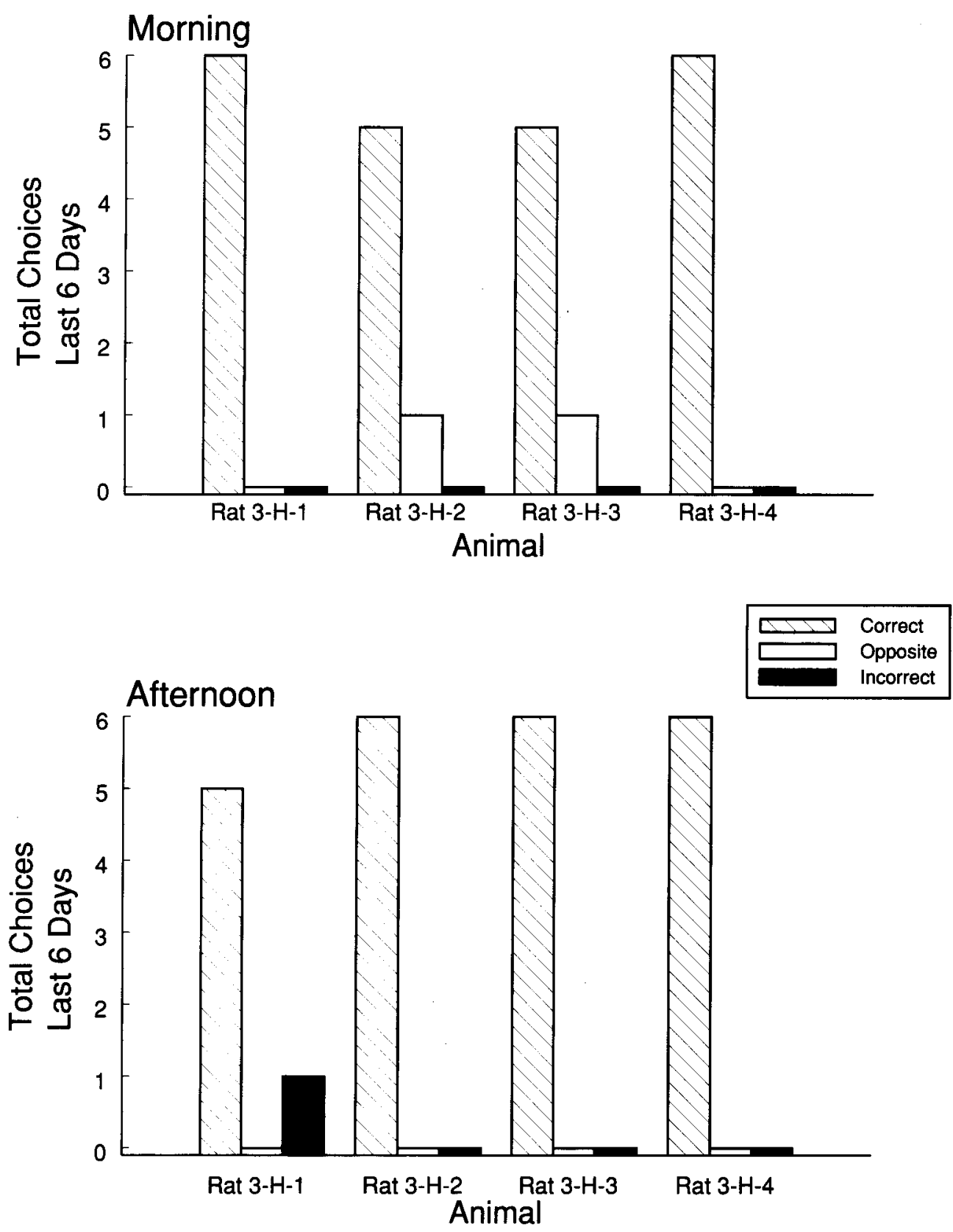

Figure 5. Total number of correct, opposite, and incorrect choices for each animal from the last 6 days of animals that climbed to the highest level in Experiment 2. The upper panel shows choices from the morning time, and the lower panel shows choices from the afternoon time. Correct choices are made to the location baited for the current daily test trial. Opposite choices are made to the location baited for the other daily test trial. Incorrect choices are made to locations that are never baited.

$51.5, p<.05]$, with the animals in the lower heights showing more opposite selections.

These results show that, on the vertical maze, response cost affected the formation of time-place discriminations. As the height of the platform was raised, a greater proportion of rats showed time-place discriminations. Conversely, the lower the platform, the more likely the animals were to form location preferences or the less likely to form time-place discriminations.

\section{GENERAL DISCUSSION}

The experiments reported here provide three important findings regarding time-place discriminations. First, 
the results extend and qualify previous results regarding time-place discriminations in rats. Second, they illustrate the importance of response cost for the formation of timeplace discriminations. Finally, the results suggest that the form of the cost may be important. We will discuss each of these separately.

With respect to previous findings regarding time-place learning, the present results demonstrate that rats will form time-place discriminations in a maze apparatus. In Experiment 1B, $66 \%$ of the animals formed time-place discriminations, and in Experiment 2, all the animals required to climb to the highest platform showed time-place discriminations. This extends the demonstrated ability of rats to form time-place discriminations to circumstances more akin to those used with songbirds (Biebach et al., 1991; Biebach et al., 1989; Krebs \& Biebach, 1989). Both form time-place discriminations in environments with distinct spatial separation between locations with no further operant response required at the food location. Importantly, although rats may be able to solve time-place problems similar to those solved by songbirds, we cannot speak directly to whether rats use similar underlying mechanisms. Carr and Wilkie $(1997,1998,1999)$ provided data that indicate that rats use different mechanisms (although see Mistlberger et al., 1996). Complicating the question is the evidence that not all songbirds use the same underlying mechanisms either (Falk, Biebach, \& Krebs, 1992).

Second, we found that rats readily solve low- to mediumeffort time--place discriminations by using location preferences plus olfactory discrimination of baited locations and the local search of previous food locations. The first and third tactics replicate the findings of both Reebs (1993) with fish and Boulos and Logothetis (1990) with rats. These investigators also observed the formation of location preferences plus local search to solve time-place problems. The ability of rats to detect the presence versus the absence of food from distal olfactory cues suggests the particular importance of attending to potential differential odor stimuli in testing for time-place learning.

As to the question of form of response cost, the importance of effort in acquiring time-place discriminations is strongly supported in this series of experiments. Although the difference in time-place discrimination between Experiments $1 \mathrm{~A}$ and $1 \mathrm{~B}$ was not conclusive, because it involved more than the manipulation of time versus effort as response cost, Experiment 2 clearly showed the positive effect of increased effort on the formation of time-place discriminations. When the response cost was relatively low, the rats tended to form location preferences. When the response cost was relatively high, the rats formed time-place discriminations. As was noted above, it also is possible that mild fear added to the response cost of climbing the vertical maze, although there was little indication of this in the choice behavior after training.

Our results also suggest that response cost, in the form of choice time or recovery time (as opposed to effort), may not be an important determinant of time-place discrimination in rats. In Experiment $1 \mathrm{~A}$, when the choice cost was the increased time to climb a $13.3-\mathrm{cm}$-high obstacle and remove the lid from a food cup and the recovery cost was a 15-min time-out in the home cage, the rats failed to form time-place discriminations. It might be argued that the increased choice time caused by the maze obstacle and the food cup lid was not long enough to have an effect. The 15-min delay for recovery from an incorrect choice, on the other hand, was very long, relative to delays that markedly affect responding in operant experiments (e.g., Wilkenfield, Nickel, Blakely, \& Poling, 1992).

However, the data of Carr and Wilkie $(1997,1998)$ appear to pose a problem for the view that time delay and/ or small to medium amounts of response effort do not produce sufficient cost to produce time-place discrimination. These authors found time-place discriminations between different walls of an operant chamber under schedule conditions that required rats to satisfy a VR 16 schedule to obtain a small amount of food. This schedule, however, was considerably greater in both time and effort than the CRF schedule of Boulos and Logothetis (1990). In addition, it is possible that their rats responded to the highest level of the VR requirement, rather than to its average. Still, neither of these considerations appears nearly so costly as the 15-min time-out in our Experiment $1 \mathrm{~B}$.

An alternative to an absolute distinction between the effects of time versus effort costs on time-place discrimination is the possibility that the critical determinant is the relation of the response cost to the animal's foraging ecology. Carr and Wilkie $(1997,1998)$ used only 10 -min foraging sessions. If rats pressed initially at the wrong location, they could have spent a considerable proportion of their session discovering the error, thereby potentially losing access to a discriminable amount of the food available in this patch. Given that rats tend to forage in patches with considerable travel time between them, one might expect them to be sensitive to the amount of food in a given patch, a result supported by laboratory findings in several feeding contexts. Studies of daily intake patterns have shown that rats regulate total meal intake, rather than meal duration, over a large range of interpellet intervals (Lucas \& Timberlake, 1988). In a related vein, rats in contrast studies have been shown to be very sensitive to reductions in reliable amounts of food (Flaherty, 1982; Pecoraro, Timberlake, \& Tinsley, 1999).

By a similar appeal to foraging ecology, it could be argued that time delays in songbirds might be a very effective promoter of time-place learning. Thus, a 280 -sec recovery delay in garden warblers (Biebach et al., 1991; Biebach et al., 1989; Krebs \& Biebach, 1989) may have affected time-place discriminations because of their higher energy budgets and concentrated foraging times. Small songbirds show a lack of tolerance for extended periods of time without access to food during a foraging bout (Caraco \& Lima, 1987). 
The possibility that form of effective cost relates to the feeding ecology of the animal is also compatible with the findings of different timing mechanisms reported by Falk et al. (1992). They reported data suggesting that the feeding ecology of two species of weaver bird influenced the type of timing mechanism used to solve a time-place problem. Weavers that were insectivorous apparently used a circadian timing mechanism, whereas granivorous weavers used a noncircadian mechanism. Reebs (1996) also speculated that the differences between species of fish in time-place discrimination might be due to feeding ecology.

Finally, our data strongly support the hypothesis that in rats the storing and/or retrieving of associations that include both time and place information requires more response effort than does the storing and/or retrieving of information regarding place alone. If rats automatically stored and retrieved both time and place information, we would expect them to use it in order to most efficiently search the maze. Instead, rats tended to rely on place information alone, except when the cost of making an incorrect choice was high. Only then did rats use information regarding both the time and the place of the food. Furthermore, other failures to produce time-place discriminations, as well as the indication that time-place discriminations may be based on noncircadian timing mechanisms (Carr \& Wilkie, 1997, 1998; Falk et al., 1992) all appear to question theories that assume that animals automatically store a simple time-date stamp related to the finding of food (Gallistel, 1990; Wilkie, 1995).

\section{REFERENCES}

Biebach, H., FalK, H., \& Krebs, J. R. (1991). The effect of constant light and phase shifts on a learned time-place association in garden warblers (Sylvia borin): Hourglass or circadian clock? Journal of Biological Rhythms, 6, 353-365.

Biebach, H., Gordij, M., \& Krebs, J. R. (1989). Time-and-place learning by garden warblers, Sylvia borin. Animal Behaviour, 37, 353-360.

Boulos, Z., \& Logothetis, D. E. (1990). Rats anticipate and discriminate between two daily feeding times. Physiology \& Behavior, 48, 523-529.

Caraco, T., \& Lima, S. L. (1987). Survival, energy budgets, and foraging risk. In M. L. Commons, A. Kacelnik, \& S. J. Shettleworth (Eds.), Quantitative analysis of behavior: Vol. 6. Foraging (pp. 1-21). Hillsdale, NJ: Erlbaum.

CARR, J. A. R., TAN, A. O., \& WilkiE, D. M. (1999). Further evidence that rats use ordinal timing in a daily time-place learning task. $B e$ havioural Processes, 48, 35-48.

CARR, J. A. R., \& WILKIE, D. M. (1997). Rats use an ordinal timer in a daily time-place learning task. Journal of Experimental Psychology: Animal Behavior Processes, 23, 232-247.

CARR, J. A. R., \& Wilkie, D. M. (1998). Characterization of the strategy used by rats in an interval time-place learning task. Journal of Experimental Psychology: Animal Behavior Processes, 24, 151-162.

CARR, J. A. R., \& WILKIE, D. M. (1999). Rats are reluctant to use circa- dian timing in a daily time-place task. Behavioural Processes, 44, 287-299.

Falk, H., BiebaCh, H., \& KreBs, J. R. (1992). Learning a time-place pattern of food availability: A comparison between an insectivorous and a granivorous weaver species (Ploceus bicolor and Euplectes hordeaceus). Behavioral Ecology \& Sociobiology, 31, 9-15.

Fantino, E., \& ABarCa, N. (1985). Choice, optimal foraging and the delay-reduction hypothesis. Behavioral \& Brain Sciences, 8, 315-330.

Fla HERTY, C. F. (1982). Incentive contrast: A review of behavioral changes following shifts in reward. Animal Learning \& Behavior, 10, 409-440.

Gallistel, C. R. (1990). The organization of learning. Cambridge, MA: MIT Press.

GouLD, J. L. (1987). Honey bees store learned flower-landing behavior according to time of day. Animal Behaviour, 35, 1579-1581.

Harrison, J. M., \& BREed, M. D. (1987). Temporal learning in the giant tropical ant, Paraponera clavata. Physiological Entomology, 12, 317-320.

Hollander, M., \& Wolfe, D. A. (1973). Nonparametric statistical methods. New York: Wiley.

Krebs, J. R., \& BiebaCH, H. (1989). Time-place learning by garden warblers (Sylvia borin): Route or map? Ethology, 83, 248-256.

LuCAS, G. A., \& TimberlaKe, W. (1988). Interpellet delay and meal patterns in the rat. Physiology \& Behavior, 43, 259-264.

Mellgren, R. L., \& Brown, S. W. (1988). Discrimination learning in a foraging situation. Journal of the Experimental Analysis of Behavior, 50, 493-503.

Mellgren, R.L, Misası, L., \& Brown, S.W. (1984). Optimal foraging theory: Prey density and travel requirements in Rattus norvegicus. Journal of Comparative Psychology, 98, 142-153.

Mistlberger, R. E., De Groot, M. H. M., Bossert, J. M., \& MarCHANT, E. G. (1996). Discrimination of circadian phases in intact and suprachiasmatic nuclei-ablated rats. Brain Research, 793, 12-18.

Pecoraro, N., Timberlake, W., \& Tinsley, M. (1999). Incentive downshifts evoke search behavior in rats (Rattus norvegicus). Journal of Experimental Psychology: Animal Behavior Processes, 25, 153167.

REEBS, S. G. (1993). A test of time-place learning in a cichlid fish. Behavioural Processes, 30, 273-282.

ReEBS, S. G. (1996). Time-place learning in golden shiners (Pisces: Cyprinidae). Behavioural Processes, 36, 253-262.

ROBERTS, W. A., \& ILERSICH, T. I. (1989). Foraging on the radial maze: The role of travel time, food accessibility and the predictability of food location. Journal of Experimental Psychology: Animal Behavior Processes, 15, 271-285.

Saksida, L. M., \& WILKIE, D. M. (1994). Time-of-day discrimination by pigeons, Columba livia. Animal Learning \& Behavior, 22, 143-154.

White, W., \& Timberlake, W. (1990). [A failure to find time-place learning in rats on a three-arm radial maze]. Unpublished data.

Wilkenfield, J., Nickel, M., Blakely, E., \& Poling, A. (1992). Acquisition of lever-press responding in rats with delayed reinforcement: A comparison of three procedures. Journal of the Experimental Analysis of Behavior, 58, 431-443.

Wilkie, D. M. (1995). Time-place learning. Current Directions in Psychological Science, 4, 85-89.

Wilkie, D. M., Saksida, L. M., Samson, P., \& Lee, A. (1994). Properties of time-place learning by pigeons, Columba livia. Behavioural Processes, 31, 39-56.

YDENBERG, R. C. (1984). Great tits and giving-up times: Decision rules for leaving patches. Behaviour, 90, 1-24.

(Manuscript received July 30, 1999; revision accepted for publication January 31,2000 .) 